\title{
Tempeh consumption enhanced beneficial bacteria in the human gut
}

\author{
${ }^{1}$ Stephanie, ${ }^{2}$ Kartawidjajaputra, F., ${ }^{2}$ Silo, W., ${ }^{1}$ Yogiara, Y. and ${ }^{1 *}$ Suwanto, A. \\ ${ }^{1}$ Faculty of Biotechnology, Atma Jaya Catholic University of Indonesia, Jalan Jenderal Sudirman 51 \\ Jakarta Selatan 12930, Indonesia \\ ${ }^{2}$ PT Nutrifood Indonesia, Kawasan Industri Pulogadung, Jalan Rawabali II No. 3, Jakarta 13920, Indonesia
}

\author{
Article history: \\ Received: 12 August 2018 \\ Received in revised form: 19 \\ September 2018 \\ Accepted: 21 September 2018 \\ Available Online: 5 October \\ 2018
}

\section{Keywords:}

Tempeh,

Gut microbiota,

Bifidobacterium,

Akkermansia muciniphila

DOI:

https://doi.org/10.26656/fr.2017.3(1).230

\begin{abstract}
It has been known that human gut microbiota modulates host health and the composition is affected by lifestyle, including the availability of dietary fiber. Tempeh, a kind of traditional fermented food from Indonesia, is rich in soluble fibers and microbial consortia. A previous study showed that tempeh could enhance IgA production and modulate gut microbiota composition in rats. Meanwhile, in human, tempeh supplementation for 16 days has increased the number of Akkermansia muciniphila significantly. Many Indonesians consume tempeh on a daily basis, but the effect of tempeh consumption for a longer period of time in human remains to be investigated. Therefore, the objective of this study was to investigate the effect of tempeh consumption in human for longer term, especially its effect in modulating gut microbiota composition. A total of sixteen (16) healthy females participated in this study. For the first 7 days, they were not allowed to consume any soy product; followed by 28 days of 100 gram of tempeh $(n=10)$, or $200 \mathrm{~mL}$ of soymilk $(\mathrm{n}=6)$ consumption. Analysis of gut microbiota showed that soymilk and tempeh supplementation generated different profiles. Twenty-eight days of tempeh supplementation seemed to improve metabolic health markers better than soymilk treatment; significantly enhancing the presence of Bifidobacterium and A. muciniphila populations compared to those at the beginning of the study.
\end{abstract}

\section{Introduction}

The gastrointestinal tract is the most denselypopulated microorganisms in the human body. Gut microbiota plays a pivotal role for human health; such as metabolic function, immune cells maturation, digesting the undigested carbohydrates, and production of shortchain fatty acid, conjugated fatty acids, exopolysaccharides, and neuroactive metabolites. Gut microbiota is also important for providing energy and bioactive compound production, such as vitamins (Patterson et al., 2016).

A study by Bressa et al. (2017) revealed that active lifestyle enhanced health-promoting bacteria. Healthy lifestyle and avoidance of excessive food intake promote better gut microbiota composition and prevent metabolic disorder (Musso et al., 2010). A previous study showed that gut microbiota in obese subjects and/or with Type-2 Diabetes were different from lean and non-diabetic subjects (Patterson et al., 2016). Obese subjects had more bacteria with carbohydrates fermentation capability than lean subjects. Lean subjects tended to have higher
Bifidobacterium (Goffredo et al., 2016), whereas nonobese subjects produced more Akkermansia muciniphila (Patterson et al., 2016).

Probiotic, prebiotic, and antibiotic therapies have improved metabolic inflammatory in mice with high-fat diet (Cani et al., 2014). A study by Wang et al. (2015) showed that probiotic addition in high-fat diet mice ameliorated gut microbiota structure to lean mice structure with an increase of Bifidobacterium production in the gut. In human study, probiotic consumption modulated human metabolism and prevented or treated diseases such as hypercholesterolemia and obesity (Fujimura et al., 2010); as well as reduce potential pathogen infection (i.e. Citrobacter and Klebsiella) and flatulence (Seo et al., 2017).

Two beneficial bacteria that would be focused in this study were Bifidobacterium and A. muciniphila. Bifidobacterium belongs to Actinobacteria phyla and commonly found in the human digestive tract. Bifidobacterium has probiotic functions, which strengthen the bowel defenses, modulate the immune 
response and exclude pathogens. Various species of Bifidobacterium that have been commonly used as probiotics include Bifidobacterium bifidum (B. bifidum), $B$. breve, $B$ lactis, and B. adolescents. Bifidobacterium species are anaerobic, rod-shaped, non-motile, and do not produce spores (Anandharaj et al., 2014). A study in human also showed that Bifidobacterium produces bile salt for lipid metabolism. The level of Bifidobacterium was known to be higher in the lean subjects compare to the obese subjects (Teixeira et al., 2013).

A. muciniphila, a mucin-degrading bacteria and is known for its properties to reduce parameters related to type-2 diabetes and obesity. A study by Dao et al. (2016) showed that calorie restriction increased the number of A. muciniphila and was associated with lower glucose level, higher insulin sensitivity, and healthier metabolic status. A. muciniphila also increased the level of endocannabinoid that controls inflammation, gut barrier, and gut peptide secretion (Everard et al., 2013).

To maintain beneficial gut microbiota composition, dietary fibers and probiotics consumption is strongly recommended. Low fiber intake is associated with increased chronic diseases, such as obesity, cardiovascular disease, type 2 diabetes, and colon cancer (Holscher, 2017).

One source of fiber-rich food is tempeh, a popular fermented food in Indonesia. As a soy product, tempeh is known for being rich in isoflavones, vitamin B-12, protein, calcium, folic acid, and easily digested due to simpler protein form after fermentation process (Babu et al., 2009). Studies revealed that Indonesian tempeh contains a consortium of microbes. Tempeh is fermented by Rhizopus microsporus var. oligosporus and contains various types of bacteria. Indonesian tempeh harbors Acetobacter indonesiensis, Klebsiella pneumoniae, Bacillus subtilis, Flavobacterium sp., Brevundimonas sp., Pseudomonas putida, and Acinetobacter spp. (Barus et al., 2008). Further metagenomics study revealed that Indonesian tempeh is inhabited by Firmicutes and Proteobacteria. There are also common Lactobacillus families, such as $L$. agilis, $L$. fermentum, and Enterobacteria cecorum (Radita et al., 2017).

A study on the Sprague Dawley rats revealed that tempeh consumption for 28 days modulated gut microbiota composition, towards a healthier gut and enhanced IgA production and IgA gene expression (Soka et al., 2014; Soka et al., 2015). Although microorganisms in tempeh were inactive due to the cooking process, they induced immune responses, such as IgA production. IgA was an antibody produced as the defense against the antigen presence in gut. A study in human showed that consumption of 100 gram of steamed tempeh per day for 16 days enhanced $\operatorname{IgA}$ production and enhanced $A$. muciniphila number significantly (Stephanie et al., 2017), which acts as improvement markers for lower diabetes type- 2 and obesity.

To our knowledge, there was no report evaluating the longer-term effect of tempeh consumption in human. This study is important because Indonesians consume tempeh on daily basis every day. Therefore, the objective of this study was to investigate tempeh consumption effect in human for longer term (28 days), specifically to its effect in modulating gut microbiota composition.

\section{Materials and methods}

\subsection{Human study}

This study was reviewed and approved by the local ethics committee, Atma Jaya Catholic University of Indonesia. The intervention followed the institution guidelines, including a written informed consent for each participant. Sixteen healthy female participants aged 2330 years old, non-smokers and not being pregnant, were divided into 2 groups. The first group comprised of 6 females, were assigned for soymilk consumption (soy group); and the second group comprised of 10 females, were assigned for tempeh consumption (tempeh group). During the intervention period, subjects were asked not to consume any probiotics or prebiotics product; and to minimize fiber consumption from fresh fruits and vegetables.

In this study, consumption of soy product was designed for 28 days adapted from the most common length of food or probiotic consumption from previous studies in human (Andreasen et al., 2012; Rampelli et al., 2012; Steenbergen et al., 2015).

For the first 7 days, participants were not allowed to consume any soy product; including soymilk, tempeh, and tofu. This treatment was intended to set a homogeneous baseline for both groups, before entering the intervention period. On the following 28 days, soy group consumed $200 \mathrm{~mL}$ soymilk per day, while tempeh group consumed 100 grams of tempeh per day. The tempeh was purchased from Empang area, Bogor, which had been previously studied (Barus et al., 2008; Soka et al., 2014; Soka et al., 2015; Stephanie et al., 2017). During the intervention, tempeh was cooked every day according to participants' preferences. However, it was strongly suggested not to cook tempeh using the deep fried method. Women were chosen as participants because of their willingness to commit to the intervention requirements neatly, especially with the cooking requirement for tempeh respondents during the intervention. Fecal samples were taken on Preliminary Measurement (PM) or 7 days before soy product 
consumption started, day 0,8 , and 29 for gut microbiota analysis (Figure 1).

\begin{tabular}{c|c|c|c|}
\cline { 3 - 4 } Consumption & $\begin{array}{c}\text { No soy product } \\
\text { consumption }\end{array}$ & $\begin{array}{c}\text { Tempeh/soymilk consumption } \\
\text { (depending on the group) }\end{array}$ \\
\cline { 2 - 4 } $\begin{array}{c}\text { Fecal } \\
\text { Collection }\end{array}$ & $\mathrm{PM}$ & 0 & 8
\end{tabular}

Figure 1. Timeline of soy/tempeh intervention and fecal collection. Preliminary measurement or PM was done 7 days before soy product consumption started.

\subsection{Total fecal bacterial DNA extraction}

Fecal samples were initially homogenized, then the fecal bacterial DNAs were extracted using QIAamp DNA Stool Mini Kit (Qiagen, Germany). Modification was performed by adding glass beads to ensure that fecal samples were homogeneous. All bacterial genomic DNAs were confirmed using $1.5 \% \mathrm{w} / \mathrm{v}$ agarose gel visualization. DNA concentration was measured with Nanodrop (Thermo Scientific, DE, USA) and diluted into $50 \mathrm{ng} / \mu \mathrm{L}$, subsequently.

\subsection{Gut microbiota enumeration}

Gut microbiota analysis was performed using realtime PCR Step One Plus ${ }^{\mathrm{TM}}$ (Applied Biosystems, CA, USA) with SYBR Green (Promega, CA, USA) as signaling detector. The standard curve was obtained using serial dilution with bacterial specific 16S rRNA gene in pGEM $^{\circledR} \mathrm{T}$ Easy (Promega, CA, USA). Isolation of DNA plasmid was performed using the Alkaline Lysis method. Isolated Plasmid was diluted into 7 points of standard concentration between $10^{6}-10^{11}$ DNA copies per reaction. Condition applied for real-time analysis consists of 1 cycle of $94^{\circ} \mathrm{C}$ for $10 \mathrm{mins}$ and 40 cycles of $95^{\circ} \mathrm{C}$ for $15 \mathrm{sec}, 60$ or $64^{\circ} \mathrm{C}$ for $30 \mathrm{sec}$, and $72^{\circ} \mathrm{C}$ for $30-$ $40 \mathrm{sec}$ depending on which gene was being detected (Table 1). All reactions were performed were duplicated.

\subsection{Food recall analysis}

Twenty-four-hour food recall data were collected on the two-weekdays period, employing phone call interview method. The difference between the two foodrecall interviews was about 2-3 weeks. All data were inputted into ESHA's Food Processor ${ }^{\circledR}$ Nutrition Analysis software (Salem, OR, USA) to obtain the number of daily calories, protein, carbohydrates, fiber, fat, and sugar. The data were analyzed statistically using paired T-test.

\subsection{Statistical analysis}

Statistical analysis was performed using GraphPad version 6.0. All data were tested for its normality distribution. Significant differences between soy group and tempeh group; and within each group were analyzed using T-test $(\mathrm{p}<0.05)$.

\section{Results}

\subsection{Food recall analysis}

For each participant, two 24-hour food recall data were compared using a paired test (data not shown). Data between soy and tempeh group were compared in the same time-point. The results showed that there was no significant difference in food pattern in both groups.

\subsection{Gut microbiota quantification}

At the beginning of the study, fecal samples were collected for preliminary measurement analysis. Our data showed that there was no significant difference in gut microbiota patterns for both soy and tempeh group at the beginning of intervention (Figure 2).

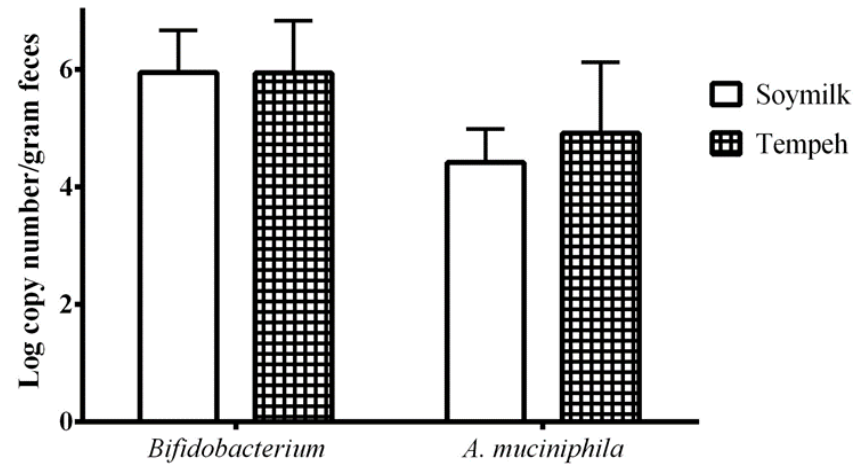

Figure 2. Gut microbiota composition for the preliminary measurement, which was measured before soy/tempeh intervention.

Enhancement of species-specific group was observed for Bifidobacterium and A. muciniphila number. In the soy group, the number of both Bifidobacterium and $A$. muciniphila decreased after 28 days of intervention. Meanwhile, in tempeh group, Bifidobacterium increased significantly after 28 days of consumption (Figure 3A), which was observed around for about 1.5-fold (Figure

Table 1. Gut microbiota primer list and analysis condition (Matsuki et al., 2004; Collado et al., 2007 )

\begin{tabular}{|c|c|c|c|c|c|}
\hline $\begin{array}{l}\text { Bacterial Group } \\
\text { Specific }\end{array}$ & Primers & Sequence & Size & Annealing $\left({ }^{\circ} \mathrm{C}\right)$ & Elongation (s) \\
\hline Akkermansia & AM1 & CAGCACGTGAAGGTGGGGAC & & & \\
\hline muciniphila & AM2 & CCTTGCGGTTGGCTTCAGAT & 327 & 64 & 40 \\
\hline Bifidobacterium & $\begin{array}{l}\text { g-Bifid-F } \\
\text { g-Bifid-R }\end{array}$ & $\begin{array}{l}\text { CTCCTGGAAACGGGTGG } \\
\text { GGTGTTCTTCCCGATATCTACA }\end{array}$ & $549-563$ & 60 & 40 \\
\hline
\end{tabular}




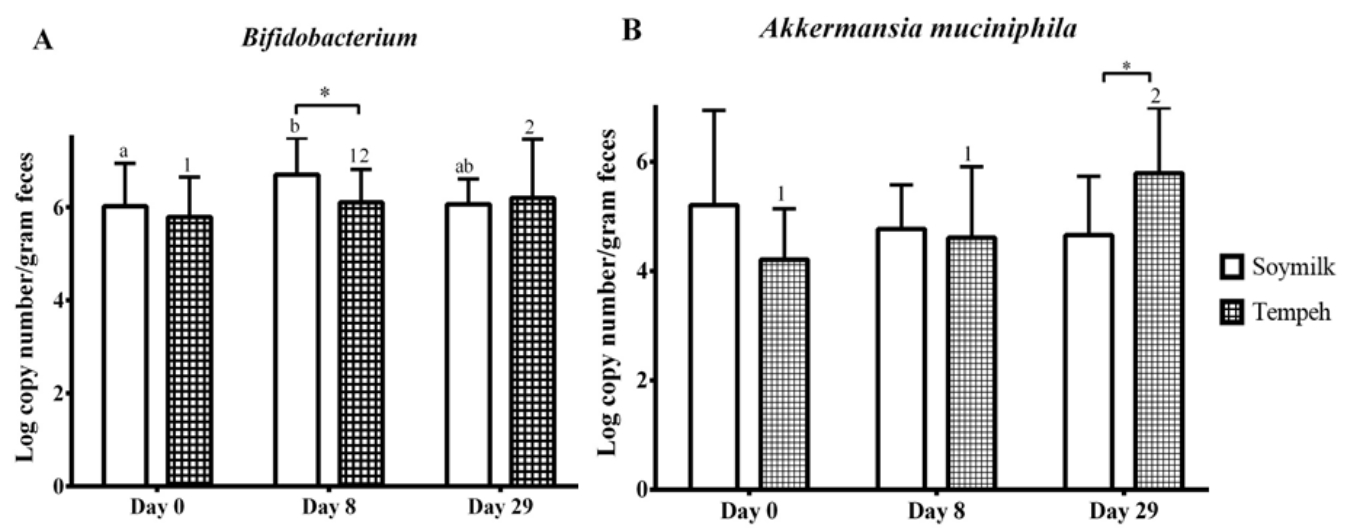

Figure 3. Gut microbiota enumeration on soy and tempeh group; (A) was for Bifidobacterium level, and (B) was for A. muciniphila level. (*) shows a significant difference between soy and tempeh group, alphabetical symbols were the significant label for each time point in soy group, and numerical symbols were the significant label for each time point in tempeh group.
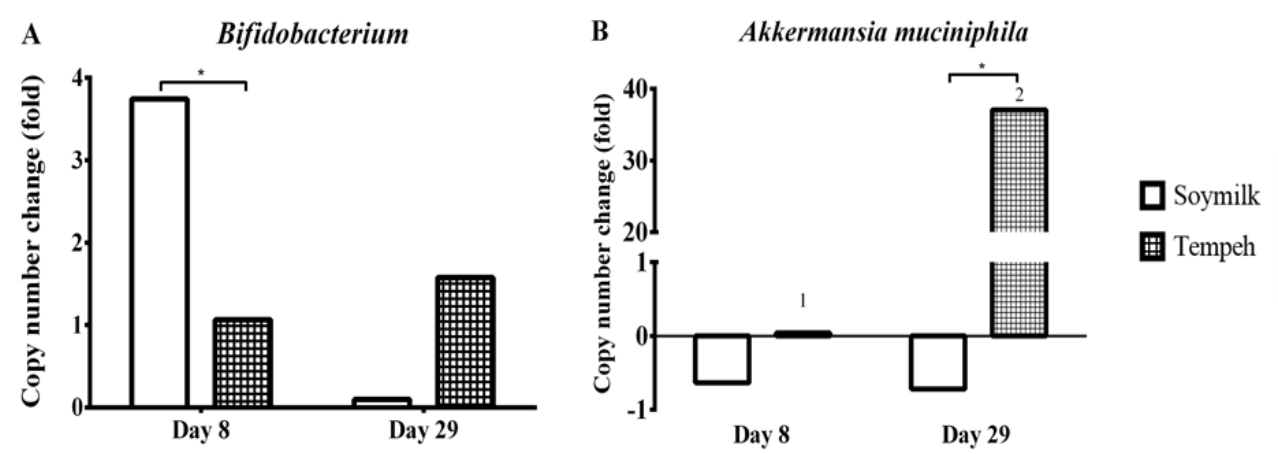

Figure 4. Gut microbiota population dynamics on day 8 and 29 compared to day 0; (A) was for Bifidobacterium, and (B) was for A. muciniphila. $\left(^{*}\right)$ shows a significant difference between soy and tempeh group, and numerical symbols were the significant label for each time point in tempeh group.

4A). It was also observed that after tempeh consumption, the number of $A$. muciniphila significantly increased compared to day 0 and day 8 (Figure 3B) for about 37fold (Figure 4B). The enhancement of $A$. muciniphila for tempeh group on day 29 was significantly different compared to soy group (Figure 3B and 4B).

\section{Discussion}

Before the intervention period, we performed a preliminary measurement to ensure the food pattern was homogeneous for both soy and tempeh group. Our result showed that there was no significant difference of macronutrient and fiber intake in both groups (data not shown); indicating that the modulation of gut microbiota during this study was mostly due to the given treatment. Besides, gut microbiota composition was also measured. There was no significant difference found between soy and tempeh group; indicating that gut microbiota composition was homogenous in all participants; thus, the condition was ideal for the start of the study.

Fecal samples were also collected on day 0,8 , and 29. Day 0 represented gut microbiota composition after 7 days of non-soy product consumption, day 8 represented gut microbiota composition after short-term soy product consumption for 7 days, and day 29 represented gut microbiota composition after longer-term consumption for 28 days.

Tempeh consumption for 28 days enhanced the production of Bifidobacterium in the gut, while soymilk consumption did not. This finding might be due to the fiber composition in tempeh and soymilk. A study by Boler et al. (2011) revealed soluble maize fiber could enhance Bifidobacterium production in healthy males. Tempeh is rich of soluble fibers (Babu et al., 2009), therefore this property might contribute to the effect of tempeh consumption in enhancing Bifidobacterium significantly.

In comparison to fermented food, whey cheese is rich of amino acids, such as threonine and cysteine. Whey cheese supplementation in mice improved metabolic markers for inflammation in the large intestine, mucus production, and fecal lactobacilli and bifidobacteria (Sprong et al., 2010). Our study indicated that tempeh consumption is promising to improve metabolic biomarkers for large intestine health and was proven already to enhance bifidobacteria population in human.

Our result showed that tempeh consumption increased A. muciniphila level for about 35-fold after 28 days of tempeh consumption, while soymilk consumption decreased the level of A. muciniphila, in 
which the lowest value was found at the end of the study (day 29). This finding was consistent with the previous study, in which 16 days of tempeh consumption increased A. muciniphila production in the human gut (Stephanie et al., 2017).

The enhancement of $A$. muciniphila production in the gut might be caused by polyphenol compound in tempeh. Soybean is a concentrated source of secondary metabolites, such as polyphenol compound (Messina 1999). The previous study revealed that dietary polyphenol from grape in high-fat diet mice lowered the expression of inflammatory markers, glucose absorption related genes, and dramatically increased $A$. muciniphila and reduced the proportion of Firmicutes to Bacteroidetes (Roopchand et al., 2015). A study by Monk et al. (2016) showed that mice fed with high-fat diet supplemented with navy bean had 20-fold higher of A. muciniphila production compared to mice fed with high-fat diet per se, which indicated that soybean solely was sufficient to enhance $A$. muciniphila in mice. Nevertheless, a significant increase of A. muciniphila after 28 days of tempeh consumption indicated that tempeh consumption gave healthier metabolic status by improving obesity and type- 2 diabetes parameters in the human gut.

Our study also showed that beneficial bacteria decreased after soymilk consumption; indicating that soluble macromolecule, such as protein, and carbohydrate in soymilk might not be sufficient to enhance beneficial bacteria. In contrast, tempeh has a higher bioavailability of macromolecules due to the fermentation process (Nout and Kiers, 2005), which seemed to enhance beneficial bacteria in the human gut.

In addition, taking into consideration that tofu is made by curdling soymilk with a coagulant, our findings indicated that beneficial bacteria might decrease after tofu consumption and enhance biomarkers for inflammation. This hypothesis was aligned with a crosssectional study by Pramudya (2015) and Hogervorst et al. (2008). A study in males revealed that the suppression of Enterococcus bacteria was lower in participants who consumed tofu rather than tempeh (Pramudya, 2015). In addition, brain-gut microbiota axis was found to trigger inflammation and metabolic diseases, such as dementia. Indonesian elderly participants with high tofu intake had worse memory compare to participants who consumed tempeh (Alkasir et al., 2017).

The enhancement of beneficial bacteria was observed in female participants, which have higher hormone fluctuations compare to male, by nature. Therefore, we hypothesized that enhancement of beneficial bacteria in male participants might develop different gut microbiota profiles.

Further study is needed to conclude whether the enhancement of beneficial bacteria was due to the bean in tempeh solely; or due to the interaction of bean and consortium of microbes inside of tempeh.

\section{Conclusion}

Taken together, tempeh and soymilk consumption gave different effects on gut microbiota composition. In general, we found that tempeh consumption modulated gut microbiota towards a healthier profile by increasing the number of Bifidobacterium and A. muciniphila significantly. These results were important as a baseline for future researches to explore the potential of tempeh in managing metabolic syndrome diseases, such as obesity and type- 2 diabetes. Furthermore, a more controlled diet for every participant is suggested, to obtain a stronger evidence of tempeh's effects in modulating human gut microbiota.

\section{Conflict of Interest}

The authors declare no conflict of interest.

\section{Acknowledgement}

This research was supported by grant from Faculty of Biotechnology, Atma Jaya Catholic University of Indonesia to Antonius Suwanto.

\section{Reference}

Alkasir, R., Li, J., Li, X., Jin, M. and Zhu, B. (2017). Human gut microbiota: the links with dementia development. Protein Cell, 8(2), 90-102. https:// doi.org/10.1007/s13238-016-0338-6

Anandharaj, M., Sivansankari, B. and Rani, R.P. (2014). Effects of probiotics, probiotics, dan synbiotics on hypercholesterolemia: a review. Chinese Journal of Biology, 2014, 1-7. https:// doi.org/10.1155/2014/572754

Andreasen, A.S., Larsen, N., Pedersen-Skovsgaard, T., Berg, R.M.G., Møller, K., Svendsen, K.D., Jakobsen, M. and Pedersen, B.K. (2010). Effects of Lactobacillus acidophilus NCFM on insulin sensitivity and the systemic inflammatory response in human subjects. British Journal of Nutrition, 104 (12), 1831-1838. https://doi.org/10.1017/ S0007114510002874

Babu, P.D., Bhakyaraj, R. and Vidhyalakshmi, R. (2009). A low cost nutritious food "tempeh"- a review. World Journal of Dairy Food Science, 4(1), 
22-27.

Barus, T., Suwanto, A., Wahyudi, A.T. and Wijaya, H. (2008). Role of bacteria in tempeh bitter taste formation: microbiological and molecular biological analysis based on 16S rRNA gene. Microbiology Indonesia, 2(1), 17-21. https://doi.org/10.5454/ mi.2.1.4

Bressa, C., Bailén-Andrino, M., Pérez-Santiago, J., González-Soltero, R., Pérez, M., MontalvoLominchar, M.G., Maté-Muñoz, J.L., Domínguez, R., Moreno, D. and Larrosa, M. (2017). Differences in gut microbiota profile between women with active lifestyle and sedentary women. PLoS ONE, 12, $\mathrm{e} 0171352$.

https://doi.org/10.1371/ journal.pone. 0171352

Cani, P.D., Geurts. L., Matamoros, S., Plovier, H. and Duparc, T. (2014). Glucose metabolism: focus on gut microbiota, the endocannabinoid system and beyond. Diabetes and Metabolism, 40(4), 246-257. https://doi.org/10.1016/j.diabet.2014.02.004

Collado, M.C., Derrien, M., Isolauri, E., de Vos, W.M. and Salminen, S. (2007). Intestinal integrity and Akkermansia muciniphila, a mucin-degrading member of intestinal microbiota present in infants, adults, and the elderly. Applied and Environmental Microbiology, 73(23), 7767-7770. https:// doi.org/10.1128/AEM.01477-07

Dao, M.C., Everard, A., Aron-Wisnewsky, J., Sokolovska, N., Prifti, E., Verger, E.O., Kayser, B.D., Levenez, F., Chilloux, J., Hoyles, L., MICROObes Consortium, Dumas, M.-E., Rizkalla, S.W., Doré, J., Cani, P.D. and Clément, K. (2016). Akkermansia muciniphila improved metabolic health during a dietary intervention in obesity: relationship with gut microbiome richness and ecology. Gut Microbes, 65(3), 426-436. https://doi.org/10.1136/ gutjnl-2014-308778

Everard, A., Belzer, C., Geurts, L., Ouwerkerk, J.P., Druart, C., Bindels, L.B., Guiot, Y., Derrien, M., Muccioli, G.G., Delzenne, N.M., de Vos, W.M. and Cani, P.D. (2012). Cross-talk between Akkermansia muciniphila and intestinal epithelium controls dietinduced obesity. Proceedings of the National Academy of Sciences, 110(22), 9066-9071. https:// doi.org/10.1073/pnas. 1219451110

Fujimura, K., Slusher, N., Cabana, M. and Lynch, S. (2010). Role of the gut microbiota in defining human health. Expert Review of Anti-Infective Therapy, 8 (4), 435-454. https://doi.org/10.1586/eri.10.14

Goffredo, M., Mass, K., Parks, M.J., Wagner, D.A., Mc Clure, E.A., Graf, J., Savoye, M., Pierpont, B., Cline, G. and Santoro, N. (2016). Role of gut microbiota and short chain fatty acids in modulating energy harvest and fat partitioning in youth. The Journal of Clinical Endocrinology and Metabolism, 101(11), 4367-4376. https://doi.org/10.1210/jc.20161797

Hogervorst, E., Sdjimim, T., Yesufu, A., Kreager, P. and Rahardjo, T.B. (2008). High tofu intake is associated with worse memory in elderly Indonesian man and women. Dementia and Geriatric Cognitive Disorders, 26(1), 50-57. https:// doi.org/10.1159/000141484

Holscher, H.D. (2017). Dietary fiber and prebiotics and the gastrointestinal microbiota. Gut Microbes, 8(2), 172-184. doi.org/10.1080/19490976.2017.1290756

Matsuki, T., Watanabe, K., Fujimoto, J., Takada, T. and Tanaka, R. (2004). Use of 16S rRNA gene-targeted group-specific primers for real-time PCR analysis of predominant bacteria in human feces. Applied Environment Microbiology, 70(12), 7220-7228. https://doi.org/10.1128/AEM.70.12.7220-7228.2004

Monk, J.M., Lepp, D., Wu, W., Graf, D., Hutchinson, A.L., Robinson, L.E. and Power, K.A. (2016). Navy bean supplementation in obesity increases Akkermansia muciniphila abundance and attenuates obesity related impairments in gut barrier function. The FASEB Journal, 30(Suppl. 1), 421.2.

Musso, G., Gambino, R. and Cassader, M. (2010). Obesity, diabetes, and gut microbiota. The hygiene hypothesis expanded? Diabetes Care, 33(10), 2277 2284. https://doi.org/10.2337/dc10-0556

Nout, M.J.R. and Kiers, J.L. (2005). Tempe fermentation, innovation, and functionality: update into the third millennium. Journal of Applied Microbiology, 98(4), 789-805. https:// doi.org/10.1111/j.1365-2672.2004.02471.x

Patterson, E., Ryan, P.M., Cryan, J.F., Dinan, T.G., Ross, R.P., Fitzgerald, G.F. and Stanton, C. (2016). Gut microbiota, obesity, and diabetes. Postgraduate Medical Journal, 92(1087), 286-300. https:// doi.org/10.1136/postgradmedj-2015-133285

Pramudya, G. (2015). Efek tempe terhadap kemunculan Enterococcus yang resisten Amoxcicillin pada feces manusia setelah konsumsi Amoxicillin-asam klavulanat. Jakarta, Indonesia: Atma Jaya Catholic University of Indonesia: BSc thesis [Bahasa Indonesia].

Radita, R., Suwanto, A., Kurosawa, N., Wahyudi, A.T. and Rusmana, I. (2017). Metagenome analysis of tempeh production: Where did the bacterial community in tempeh come from? Malaysian Journal of Microbiology, 13(4), 280-288.

Rampelli, S., Candela, M., Severgnini M., Biagi E., 
Turroni S., Roselli, M., Carnevali, P., Donini, L. and Brigidi, P. (2013). A probiotics-containing biscuit modulates the intestinal microbiota in the elderly. The Journal of Nutrition, Health, and Aging, 17(2), 166-172. https://doi.org/10.1007/s12603-012-0372-x

Roopchand, D.E., Carmody, R.N., Kuhn, P., Moskal, K., Rojas-Silvia, P., Turnbaugh, P.J. and Raskin, I. (2015). Dietary polyphenols promote growth of the gut bacterium Akkermansia muciniphila and attenuate high-fat diet-induced metabolic syndrome. Diabetes, 64(8), 2847-2858. https://doi.org/10.2337/ db14-1916

Seo, M., Heo, J., Yoon, J., Kim, S.-Y., Kang, Y.-M., Yu, J. and Cho, S. (2017). Methanobrevibacter attenuation via probiotic intervention reduces flatulence in adult human: A non-randomised paireddesign clinical trial of efficacy. PLoS ONE, 12(9), $\mathrm{e} 0184547$. https://doi.org/10.1371/ journal.pone. 0184547

Soka, S., Suwanto, A., Sajuthi, D. and Rusmana, I. (2014). Impact of tempeh supplementation on gut microbiota composition in Sprague-Dawley rats. Research Journal of Microbiology, 9(4), 189-198. https://doi.org/10.3923/jm.2014.189.198

Soka, S., Suwanto, A., Sajuthi, D. and Rusmana, I. (2015). Impact of tempeh supplementation on mucosal immunoglobulin A in Sprague-Dawley Rats. Food Science and Biotechnology, 24(4), 14811486. https://doi.org/10.1007/s10068-015-0191-z

Sprong, R.C., Schonewille, A.J. and van der Meer, R. (2010). Dietary cheese whey protein protects rats against mild dextran sulfate sodium-induced colitis: role of mucin and microbiota. Journal of Dairy Science, 93(4), 1364-1371. https://doi.org/10.3168/ jds.2009-2397

Steenbergen, L., Sellaro, R., van Hemert, S., Bosch, J.A. and Colzato, L.S. (2015). A randomized controlled trial to test the effect of multispecies probiotics on cognitive reactivity to sad mood. Brain, Behavior, and Immunity, 48, 258-264. https://doi.org/10.1016/ j.bbi.2015.04.003

Stephanie, Ratih, N.K., Soka, S. and Suwanto, A. (2017). Effect of tempeh supplementation on the profiles of human intestinal immune system and gut microbiota. Microbiology Indonesia, 11(1), 11-17. https:// doi.org/10.5454/mi.11.1.2

Teixeira, T.F.S., Grzeskowiak, L.M., Salminen, S., Laitinen, K., Bressan, J. and Peluzio, M.D.C.G. (2013). Fecal levels of Bifidobacterium and Clostridium coccoides but not plasma lipopolysaccharide are inversely related to insulin and HOMA index in women. Clinical Nutrition, 32
(6),
1017-1022.
https://doi.org/10.1016/

j.clnu.2013.02.008

Wang, J., Tang, H., Zhang, C., Zhao, Y., Derrien, M., Rocher, E., Vlieg, J.E.V.-H., Strissel, K., Zhao, L., Obin, M. and Shen, J. (2015). Modulation of gut microbiota during probiotic-mediated attenuation of metabolic syndrome in high fat diet-fed mice. The ISME Journal, 9(1), 1-15. https://doi.org/10.1038/ ismej.2014.99 